\title{
Robust Single Polarization Coherent Transceiver Using DGD Pre-distortion for Optical Access Networks
}

\author{
Md. Saifuddin Faruk ${ }^{1}$, Hadrien Louchet ${ }^{2}$, and Seb. J. Savory ${ }^{1}$ \\ ${ }^{l}$ Electrical Engineering Division, Engineering Department, University of Cambridge, Cambridge CB3 OFA, U.K. \\ ${ }^{2}$ VPIphotonics GmbH, Berlin10587, Germany \\ ․msf35@,cam.ac.uk
}

\begin{abstract}
DGD pre-distortion implements a polarization time code enabling a single polarization coherent transceiver to recover the signal for all polarization states. Using a DFE the maximum polarization dependent sensitivity variation is $1.9 / 3.0 \mathrm{~dB}$ for BPSK/QPSK respectively.

OCIS codes: (060.2330) Fiber optics communications; (060.1660) Coherent communications; (060.2840) Heterodyne.
\end{abstract}

\section{Introduction}

The next generation optical access networks are expected to provide gigabits/s data rate per user, the long reach of 60 to $100 \mathrm{~km}$ and a high splitting factor of up to 1000 . Coherent technology is the feasible solution to fulfill all these requirements $[1,2]$. However, given the cost constraints of access networks, this must be attained by means of very simple electronic and photonic devices.

Recently a simple single-polarization heterodyne receiver consisting of a 3-dB coupler and a single balanced photodiode has been demonstrated without any optical polarization controller [3]. The polarization-insensitive operation of such receiver was achieved by using the Alamouti space-time block coding (STBC) at the transmitter [4]. However, Alamouti STBC system has the drawback that a full dual-polarization transmitter with $2 \times 2$ multipleinput multiple-output (MIMO) digital signal processing (DSP) at the transceiver are required albeit a single polarization signal is detected.

To avoid such drawbacks, in this paper, a differential-group-delay (DGD) pre-distortion based singlepolarization transmitter is demonstrated that enables using the very simplified coherent receivers without any optical polarization tracking. A conventional single polarization transmitter is used with an additional DGD pre-distortion unit that delays signal in one of the polarizations by a symbol period. Such an approach depolarizes the output signal which can be always detected by the single-polarization receiver irrespective of the change in polarization state during fiber propagation. The original signal can be restored by using a proper DSP algorithm at the receiver. A decision-feedback equalizer (DFE) is proposed for such purpose. The performance of the proposed scheme is evaluated by means of simulation for 12-Gbits/s (considering 10-Gbits/s downlink channel with 20\% FEC overhead) BPSK and QPSK signal transmission over 100-km standard single-mode fiber (SMF).

\section{DGD pre-distortion aided single-polarization coherent transceiver}

\subsection{System description}

The block diagram for the proposed DGD pre-distortion based downstream transmitter is shown in Fig. 1(a). The DGD pre-distortion is introduced by splitting the signal from a conventional single-polarization transmitter using a polarization beam splitter (PBS), a fiber delay of one symbol period in one polarization and then combining the signal by polarization beam combiner (PBC). Since the $x$ - and $y$-polarization carry the same signal, with just a symbol-period delay between them, no information is lost whether $x$ - or $y$-polarization is detected and thus data can be recovered by the DSP in the receiver. Now if one of the polarization modes, say, $x$-polarization signal is detected by the simplified single-polarization heterodyne receiver as shown in Fig. 1(b), the received signal can be written as-

$$
S_{x, k}^{\prime}=\left(h_{x x} S_{k}+h_{x y} S_{k-1}\right) e^{j \theta_{n}, k}+n_{k} .
$$

where, $S_{k}$ is the data symbol, $\theta_{k}$ is the phase noise, and $n_{k}$ is the additive white Gaussian noise (AWGN) and $h_{x x}$ and $h_{x y}$ are the elements of Jones matrix $H=\left[\begin{array}{lll}h_{x x} & h_{x y} ; h_{y x} & h_{y y}\end{array}\right]$ that represents the polarization states.

Therefore, the received signal can be considered as a filtered version of the transmitted signal by a transfer function of $H_{p o l .}(z)=h_{x x}+h_{x y} z^{-1}$ due to the state of polarization. For polarization tracking in the receiver DSP, we have to produce $w(z)$ such that

$$
w(z)=\frac{1}{H_{p o l .}(z)}=\frac{1}{h_{x x}+h_{x y} z^{-1}} .
$$



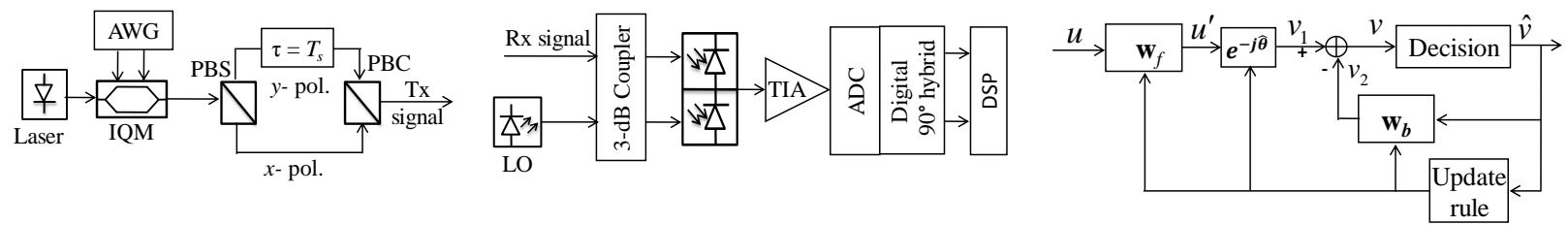

Fig.1. Proposed system configuration. (a): Symbol-period DGD distorted transmitter; (b): Simplified heterodyne receiver; and (c): The DFE configuration for joint equalization, polarization tracking and carrier phase recovery in the receiver DSP.

\subsection{Digital signal processing}

As shown in Eq. (2), it is difficult to implement $w(z)$ in a feed-forward finite-impulse-response (FIR) configuration, because the length of FIR filter depends on the value of $h_{x x}$ and $h_{x y}$. For example, if $h_{x x}=1$ and $h_{y y}=0, w(z)$ can be implemented with just 1-tap (best-case), whereas when $h_{x x}=h_{x y}$, theoretically a FIR filter with an infinite number of taps is required (worst-case). However, $w(z)$ can be implemented with 2-taps infinite-impulse-response (IIR) filters. Therefore, in this work a decision-feedback equalizer (DFE) as shown in Fig. 1(c) is used which can be considered as a simple form of adaptive IIR filter. If we consider $\mathbf{w}_{f}$ and $\mathbf{w}_{b}$ as the feedforward and feedback filter tap coefficient vectors, respectively and $\hat{\theta}$ is a phase estimator, the output of the circuit can be written as

$$
v_{k}=\mathbf{w}_{f} \mathbf{u} e^{-j \hat{\theta}}-\mathbf{w}_{b} \hat{\mathbf{v}},
$$

where $\hat{\mathbf{v}}$ is the decided symbols from the output $\boldsymbol{v}$. Then the error signal for the filter adaptation using the leastmean-square (LMS) algorithm is calculated as $\varepsilon=d-v$, where $d$ is the training symbols for initial reliable convergence and $\hat{v}$ in the steady-state decision-directed (DD) mode. Consequently, the phase estimator and filters tap-weights are updated as [5]:

$$
\begin{gathered}
\hat{\theta} \leftarrow \hat{\theta}-\mu_{p} \Im\left\{\varepsilon v^{*}\right\}, \\
\mathbf{w}_{b} \leftarrow \mathbf{w}_{b}-\mu \varepsilon \hat{\mathbf{v}}^{*}, \\
\mathbf{w}_{f} \leftarrow \mathbf{w}_{f}+\mu \varepsilon e^{j \hat{\theta} \mathbf{u}^{*}},
\end{gathered}
$$

where, $\mu_{p}$ and $\mu$ are the step-size parameters.

\section{Simulation investigations}

\subsection{Simulation setup}

To verify the principle of the proposed schemes, we conduct computer simulation for a 12-Gbits/s BPSK and QPSK transmission systems over 100-km standard SMF. The signal is generated according to the transmitter configuration of Fig. 1(a). A pulse shaping filter having a root-raised cosine (RRC) profile with a roll-off factor of 0.1 is applied. The laser phase noise corresponds to a combined linewidth symbol duration product of $1 \times 10^{-4}$ is considered. The signal is then passed through the fiber whose transfer function is assumed as

$$
H_{f}=e^{-j \omega^{2} \beta_{2} z / 2}\left[\begin{array}{cc}
\cos \theta & \sin \theta \exp (-i \phi) \\
-\sin \theta \exp (i \phi) & \cos \theta
\end{array}\right],
$$

where, $\omega$ is the angular frequency of the optical carrier, $\beta_{2}$ is the group-velocity dispersion (GVD) parameter, $z$ is the fiber link length, and $2 \theta$ and $\phi$ are the azimuth and elevation rotation angles between two polarization states, respectively. Then AWGN noise is added and the signal is received by the heterodyne receiver shown in Fig. 1(b). Two-fold oversampled sequences are then passed through the RRC matched filter and the proposed DFE of Fig. 1(c). The number of taps used for $\mathbf{w}_{f}$ and $\mathbf{w}_{b}$ are 21 and 5, respectively. Finally, the symbols are differentially decoded and bit-error rate (BER) is measured by direct counting method.

\subsection{Results and discussion}

To evaluate the tolerance of the proposed system to the polarization changes over the entire Poincare sphere, the parameters $\theta$ and $\phi$ of Eq. (7) are swept ranging $-90^{\circ}$ to $90^{\circ}$. We include a 2-dB (for BPSK) or 3.25-dB (for QPSK) signal-to-noise ratio (SNR) margin from that required for a BER of $1.5 \times 10^{-2}\left(20 \%\right.$ HD-FEC limit) and $\Delta v T_{s}$ of $1 \times 10^{-4}$. The BER fluctuation for such two-dimensional sweep is shown in Fig. 2. The BER performance is found 

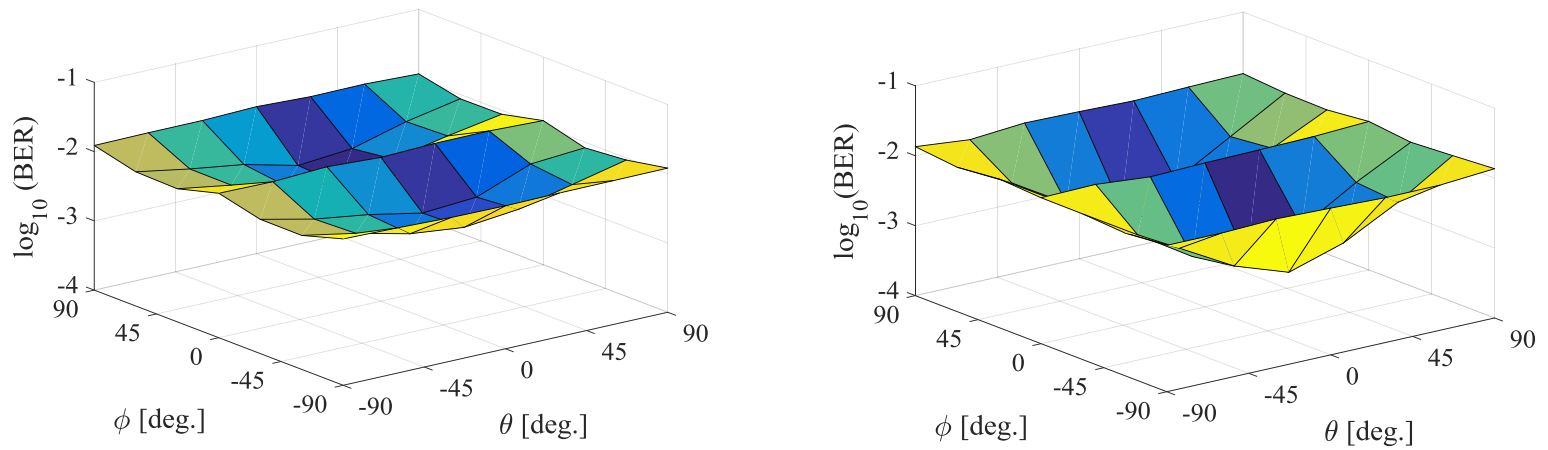

Fig. 2. Variation of BER with different polarization state swept over full Poincare sphere for (a) BPSK and (b) QPSK system.

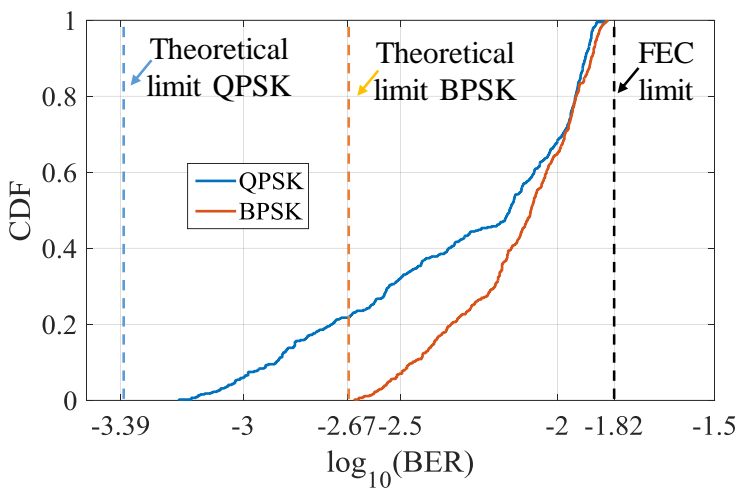

Fig. 3. Cumulative density function (CDF) of $\log _{10}(\mathrm{BER})$ from 440 different polarization states.

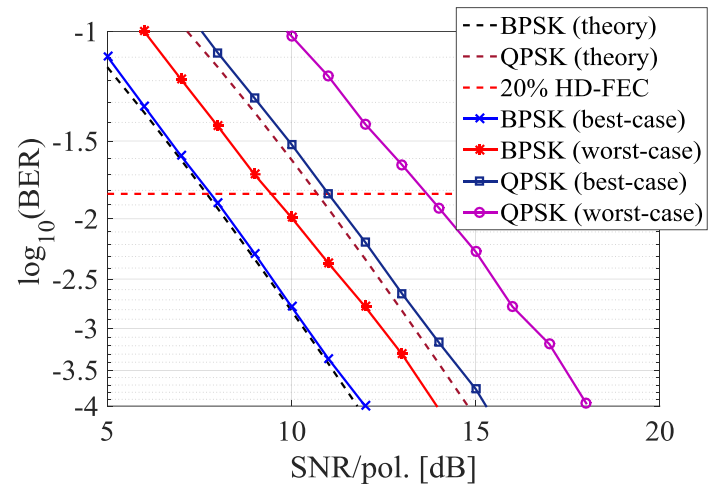

Fig. 4. The BER performance of the prosed system as a function of SNR per polarization.

dependent on SOP of the incoming signal. This is because the characteristics and performance of the digital filters depend on the change of polarization states as described in Sec. 2. To quantify the dependence, cumulative density function (CDF) of BER is calculated from 440 different polarization states swept over full Poincaré sphere. It is found that for both the modulation formats the worst-case BER is always below the FEC limit while the best-case BER is very close to the theoretical limit. Finally, we evaluate the BER performance as a function of SNR per polarization as shown in Fig. 4. It is found that worst-case SNR penalty at BER of 1.5\% due to polarization rotation for BPSK and QPSK systems are around 1.9 and $3 \mathrm{~dB}$, respectively.

\section{Conclusion}

We have proposed a low complexity and robust single-polarization coherent transceiver based on polarization time coding using DGD pre-distortion and heterodyne detection; aiming at its application for optical access networks. A DFE suitable for such system has been developed that enables data recovery within a reasonable sensitivity variation for all polarization states of the received signal.

\section{Acknowledgement}

This work was partly supported by EU project ICONE, grant no. 608099 .

\section{References}

[1] S. J. Savory, "Digital coherent optical access networks," in IEEE Photonics Conference (IPC), 125-126 (2013).

[2] D. Lavery, R. Maher, D. S. Millar, B. C. Thomsen, P. Bayvel, and S. J. Savory, "Digital coherent receivers for long-reach optical access networks," J. Lightwave Technol. 31, 609-620 (2013).

[3] M. S. Erkılınç, D. Lavery, K. Shi, B. C. Thomsen, P. Bayvel, R. I. Killey, and S. J. Savory, "Polarization-insensitive single-balanced photodiode coherent receiver for long-reach WDM-PONs," J. Lightwave Technol. 34, 2034-2041 (2016).

[4] S. M. Alamouti, "A simple transmit diversity technique for wireless communications," IEEE J. Sel. Areas Commun. 16, 1451-1458 (1998).

[5] I. Fatadin, D. Ives, and S. J. Savory, "Blind equalization and carrier phase recovery in a 16-QAM optical coherent system," J. Lightwave Technol. 27, 3042-3049 (2009). 